formin has not decreased (1.63/million DDDs in 1972-4 and $1 \cdot 76 /$ million DDDs in 1975-7).

Previous studies have shown that about $30 \%$ of serious ADRs to drugs were reported to the Swedish committee. ${ }^{5}$ Although the total number of reports has increased since its inception in 1965, reports of death have been fairly constant. ${ }^{5} 8$ This has been interpreted as showing that serious reactions, especially fatal ones, are overrepresented in the reports. The total numbers of reports of ADRs to metformin and phenformin are of the same order when related to use (table III), which speaks against a serious reporting bias. Furthermore, age and sex aistributions in the prescription sample were similar for the two drugs, as were the prescribed daily doses versus the respective DDDs. Metformin is eliminated by the kidneys while phenformin is also metabolised. ${ }^{1}$ Accordingly, the daily dose of phenformin and the age of patients receiving metformin seem to be important factors in the development of ADRs to the drugs. An age limit of 60 years for biguanide use would have eliminated $70 \%$ of the patients treated (figure) and 78\% (40/51) of the cases of lactic acidosis classified as probable or not excluded. The youngest patient with lactic acidosis was only 20 years old.

Our findings indicate that this methodology is valuable for comparing the incidences of ADRs to similar drugs. Since ADRs are underreported, ${ }^{5}$ however, these figures should not be extra- polated to "true" incidences. Our study also indicates that phenformin is more likely than metformin to cause lactic acidosis. This is a clinical advantage for metformin under the premise that both drugs are equally effective.

This study was supported by a grant from the Karolinska Institutet. $\frac{1}{1}$ We are indebted to the National Corporation of Swedish Pharmacies $\cong$ for providing material from their prescription survey. We also thank $\subseteq$ $\mathrm{Mr}$ Staffan Ekblom, of the statistical research group, Stockholm $\overrightarrow{\vec{F}}$ University, for evaluating the statistical methods used.

\section{References}

1 Alberti, K G M M, and Nattrass, M, Lancet, 1977, 2, 25.

2 Natrass, M, et al, Diabetologia, 1977, 13, 145.

3 Alexander, W D, and Marples, J, Lancet, 1977, 1, 191.

Bergman U, in Drug Utilization Studies Methods and Applications, ed U Bergman, A Grimsson, and B Westerholm. Copenhagen, World Health Organisation, Regional Office for Europe. In press.

${ }^{5}$ Böttiger, L E, et al, fournal of Clinical Pharmacology, 1974, 14, 401.

${ }^{6}$ Kristoferson, K, and Wessling, A, Svensk Farmaceutisk Tidskrift, 1977, 윽 $\mathbf{8 1}, 309$.

7 Luyckx, A, et al, fournées Annuelles de Diabetologie Hôtel-Dieu, 1974, p 129. స̃

8 Böttiger, L E, Furhoff, A-K, and Holmberg, L, Läkartidningen, 1977, 74, 1182 .

(Accepted 26 May 1978)

\title{
Spontaneous milk ejection during lactation and its possible relevance to success of breast-feeding
}

\author{
ALAN S MCNEILLY, JUDITH R MCNEILLY
}

British Medical fournal, 1978, 2, 466-468

\section{Summary and conclusions}

In a woman suckling twins it became apparent that both suckling-induced and precisely timed, spontaneous bursts of milk ejection were occurring. Observations on days 14, 28, 56, and 112 of lactation disclosed highly significant increases in intervals between episodes of spontaneous milk ejection. Furthermore, at all stages of lactation the interval between a feed and the next episode of spontaneous ejection was significantly longer than the interval between spontaneous ejections.

The decrease in frequency of episodes of spontaneous milk ejection during lactation may be related to the decreasing release of prolactin in response to suckling. Spontaneous milk-ejection episodes are felt only when the breast is full and may signal its readiness for a further suckling episode. Such bursts of milk ejection may stimulate the suckling response in babies, suggesting that rigid three- or four-hour feeding regimens may be unphysiological and pose a threat to the success of breastfeeding in the early postnatal period.

MRC Unit of Reproduction Biology, Edinburgh EH1 2QW ALAN S MCNEILLY, PHD, research scientist JUDITH R MCNEILLY, PHD, physiologist

\section{Introduction}

In recent years there has been a resurgence of interest in breast feeding. An increasing number of women start to breast-feed their babies, but while many continue to breast-feed for severals months a large proportion stop within a few weeks of leaving hospital. ${ }^{2}$ The reasons for stopping breast-feeding are many윽 and varied but we do not know how often it is due to failure or: inadequacy of normal physiological mechanisms. ${ }^{2}$ Lactationo comprises two separate physiological mechanisms-namely, milk secretion, which is controlled by prolactin released from? the anterior pituitary in response to the suckling stimulus; andN milk ejection, a neuroendocrine reflex in which oxytocin released from the posterior pituitary in response to sucklingo causes contraction of the alveoli of the breast and ejection of milk via the mammary ducts and nipple. Whereas the role of prolactin in lactation has received much attention, ${ }^{3-5}$ that of $\omega$ oxytocin and the factors controlling milk ejection have been? virtually ignored..$^{6-10}$

Milk ejection during suckling may be inhibited by both physical and psychological stress, and women are more ${ }^{+}$ susceptible to these stresses during early lactation. ${ }^{11}$ Newton and ${ }_{\vec{O}}$ Newton's ${ }^{11}{ }^{12}$ classical study also showed that successful breast- $\frac{0}{\mathbb{D}}$ feeders exhibit a greater milk-ejection response to suckling than $\frac{\Phi}{\Phi}$ unsuccessful breast-feeders, again emphasising the importance of oxytocin release in the success of breast-feeding.

Oxytocin release occurs in response not only to stimulation of the nipple during suckling ${ }^{8}{ }^{9}$ but also to other factors associated with breast-feeding - for example, the cry of the baby. ${ }^{710}$ In addition, in anaesthetised rats, whose young are continually on? the nipples, oxytocin release appears to occur spontaneously at precisely timed intervals independent of any suckling stimulus; the pups suckle only after spontaneous milk ejection has 
Mean intervals ( $\pm S E$ of mean) between episodes of spontaneous milk ejection and between breast-feeding and next spontaneous milk ejection, and numbers of feeding periods and suckling-induced milk ejections in 24 hours. (Observations on woman breast-feeding twins)

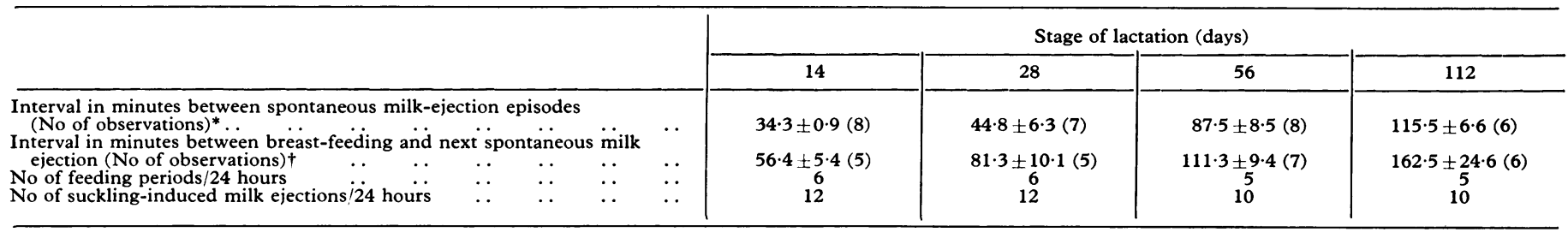

*Increase in time at each stage of lactation differs significantly $(0.05>P>0.001)$ from previous interval. IInterval to first milk ejection after breast-feeding is significantly greater $(\mathbf{P}<0.01)$ than between spontaneous milk ejections and increases significantly $(P<0.01)$ as lactation

occurred. ${ }^{13}$ This phenomenon has not been reported in other species. During normal lactation in a woman who was suckling twins it became apparent that both suckling-induced and precisely timed spontaneous milk ejections were occurring. We describe these findings and discuss their relevance to the management of mothers who wish to breast-feed.

\section{Observations}

We made our observations during the third lactation of a normal, healthy woman after she had given birth to twins. She had successfully breast-fed her first and second children for nine and seven months. The twins were fed on demand, one at $a^{*}$ time; each was allowed to suckle one breast only, the babies changing sides at each feed. Throughout the study period each baby suckled the breast for a mean of $13 \pm$ SD 3 minutes (20 observations) at a time, so that both babies were fed in 30 minutes. During every feed, for each twin milk letdown occurred 10-30 seconds after the start of suckling. In addition, however, episodes of spontaneous milk ejection occurred at predictable intervals between feeds, the sensation being identical with the mammary response experienced during feeding. This spontaneous milk ejection was never associated with any stimulus related to the babies. While some leakage of milk occurred from the unsuckled breast in response to the first suckling episode of a feed, no such leakage occurred from the first breast when the second twin was being fed, nor during the majority of spontaneous milk ejections.

The timing of suckling-induced and spontaneous milk ejection was recorded over 48 hours on the $14 \mathrm{th}, 28 \mathrm{th}, 56 \mathrm{th}$, and $112 \mathrm{th}$ days of lactation (table and figure). Spontaneous milk ejection occurred at

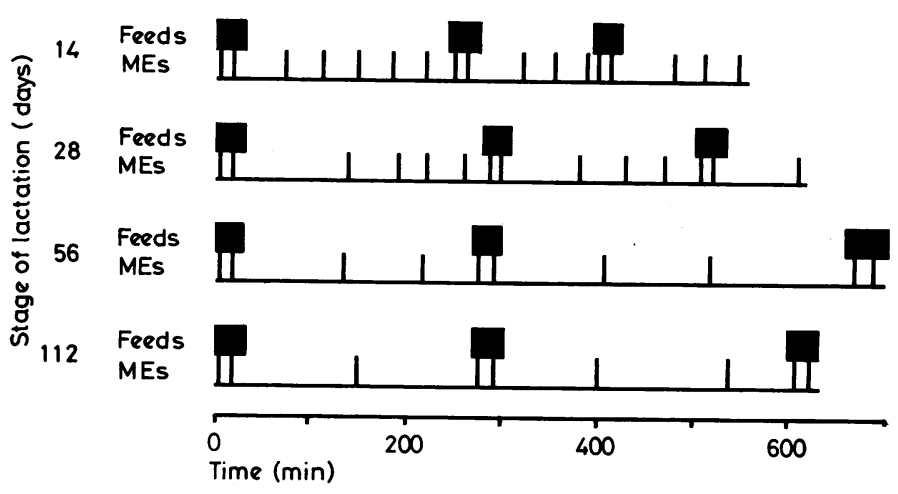

Intervals between feeds, milk ejections (MEs) associated with suckling, and spontaneous milk ejections at different stages of lactation in woman breast-feeding twins.

precise and regular intervals, which increased from $34.3 \pm \mathrm{SE}$ of mean 0.9 minute on day 14 to $115.5 \pm 6.6$ minutes on day 112 ; this increase was highly significant at each stage of lactation $(P<0.001)$. Two suckling-induced milk ejections were observed at each feeding time, and an episode of breast-feeding delayed the onset of the next spontaneous milk ejection by intervals increasing from $22 \cdot 1$ minutes on day 14 to 47.0 minutes on day 112 . Thus the time to first spontaneous milk ejection after a feed was significantly longer $(P<0.01)$ than the interval between spontaneous milk ejections at all stages of lactation. Possibly the double milk ejection occurring during the feeding period depletes the releasable pool of oxytocin or desensitises the hypothalamus or higher centres controlling milk release. On several occasions, however, suckling-induced milk ejection was induced only 10-15 minutes after spontaneous milk ejection (see figure). Some degree of breast distension was necessary for the mother to have the sensation of milk ejection, since she felt this only in the full breast when the second twin started suckling. (This accords with observations on rats, in which spontaneous milk ejection occurred only when there was distension of the mammary glands. ${ }^{13}$ ) Hence she may have had spontaneous milk ejection shortly after feeding but did not detect it owing to the lack of distension.

\section{Comment}

The decrease in frequency of spontaneous milk ejection as lactation progresses, which has not been observed in rats (D W Lincoln, personal communication), may be related to the decreasing release of prolactin in response to suckling. ${ }^{3}$ Whether prolactin is released during these spontaneous milk-ejection episodes is not known. There is no evidence of prolactin release during lactation in response to any stimuli other than tactile nipple stimulation. ${ }^{4}$

The subjective observations reported here suggest that in addition to suckling-induced releases of oxytocin women have precisely timed spontaneous releases not unlike the presumptive episodic releases of luteinising hormone-releasing hormone during the menstrual cycle. ${ }^{14}$ These spontaneous milk ejections are felt only when the breast is full, suggesting that there may be a neuroendocrine effect whereby the breast indicates its readiness for a further suckling episode. In so-called primitive societies women habitually sleep with the baby on the breast all night and carry it on the breast for much of the day. ${ }^{15} 16$ Possibly these spontaneous bursts of milk ejection are the stimulus for the suckling response in the baby, who would therefore feed at intervals of 30-60 minutes during early lactation. This may mean that the rigid three- or four-hourly feeding regimens followed in many hospitals are unphysiological and as such may pose a threat to the initial success of breast-feeding. Milk secretion depends on the amount of prolactin released in response to suckling. ${ }^{31718}$ Thus an inadequate suckling stimulus will ultimately result in a reduced secretion of milk. ${ }^{17}$ Since the mother's anxiety, often created by fear of being unable to lactate, will itself inhibit milk ejection, clearly she should have more psychological support. Moreover, advice on the optimal frequency of suckling should take account of physiological considerations. There is little value in having an adequate supply of milk in the breast if it is not made available to the hungry baby at the right moment.

We thank Professor R V Short and Drs D W and G A Lincoln for helpful discussions during this study.

\section{References}

1 West, C, unpublished observations, 1978

2 Eastham, E, et al, British Medical fournal, 1976, 1, 1467.

3 Tyson, J E, and Friesen, H G, American fournal of Obstetrics and Gynecology, 1973, 116, 377. 
${ }^{4}$ Frantz, A G, Kleinberg, D L, and Noel, G L, Recent Progress in Hormone Research, 1972, 28, 527.

5 McNeilly, A S, fournal of Biosocial Science, 1977, suppl No 4, p 5.

${ }^{6}$ Cobo, E, et al, American fournal of Obstetrics and Gynecology, 1967, 97, 519.

7 Caldeyro-Barcia, $\mathrm{R}$, in Lactogenesis: The Initiation of Milk Secretion at Parturition, ed M Reynolds and S J Folley, p 229. Philadelphia, University of Pennsylvania Press, 1969.

8 Vorherr, H, in The Breast: Morphology, Physiology and Lactation. New York, Academic Press, 1974.

${ }^{9}$ Fox, C A, and Knaggs, G S, fournal of Endocrinology, 1969, 45, 145.

10 Cobo, E, in Lactogenic Hormones, Fetal Nutrition and Lactation, ed J B Josimovich, M Reynolds, and E Cobo, p 433. New York, John Wiley and Sons, 1974.
11 Newton, N, and Newton, M, fournal of Pediatrics, 1948, 33, 698.

12 Newton, N, and Newton, M, Pediatrics, 1950, 5, 726.

13 Lincoln, D W, Hill, A, and Wakerley, J B, fournal of Endocrinology, 1973, $57,459$.

${ }^{14}$ Midgley, A R, jun, and Jaffe, R B, fournal of Clinical Endocrinology and Metabolism, 1971, 33, 962.

15 Short, R V, Proceedings of the Royal Society, Series B, 1976, 195, 3.

16 May, R M, Nature, 1978, 272, 491.

17 Aono, T, et al, fournal of Clinical Endocrinology and Metabolism, 1977, 44, 1101

${ }^{18}$ Delvoye, P, et al, Clinical Endocrinology, 1977, 7, 257.

(Accepted 2 fune 1978)

\title{
Measurement of capillary blood glucose in filter-paper spots: an aid to the assessment of diabetic control
}

\author{
K WAKELIN, D J GOLDIE, M HARTOG, A P ROBINSON
}

British Medical fournal, 1978, 2, 468-469

\section{Summary and conclusions}

A method of measuring glucose in capillary blood spotted on to filter paper was evaluated. Between-batch reproducibility was about $6 \%$, and the glucose remained stable in the spots for up to four days. Adoption of the method should improve control of diabetes, particularly insulin-dependent forms.

\section{Introduction}

The importance of close control of diabetes in reducing the incidence of complications is now widely accepted ${ }^{1}$ and has led to a re-examination of methods used for assessing control. Although many metabolic abnormalities are found in diabetics, tests reflecting blood glucose concentrations are still the most useful indicators of control. ${ }^{2}$ Traditionally the degree of hyperglycaemia has been assessed indirectly by semi-quantitative measurement of sugar in the urine. This is not sensitive enough, however, as glycosuria usually appears only when blood glucose values are very high. The concentration of circulating glycosylated haemoglobin may provide the best overall guide to the quality of diabetic control, ${ }^{3}$ but the day-to-day monitoring of insulin requirements would probably be best achieved by serial blood sugar determinations performed on patients during their normal daily activities. In some clinics patients take such samples themselves by finger-prick into tubes containing dried anticoagulant and fluoride for later glucose determination in the laboratory. We have not been successful with this approach, as most capillary blood samples clotted and were unsuitable for analysis. Sönksen et $a l^{4}$ and Walford $e t a l^{5}$ reported their experiences of self-monitoring of capillary blood glucose by

\footnotetext{
Department of Clinical Chemistry, Southmead Hospital, Bristol BS10 5NB

$\mathrm{K}$ WAKELIN, MB, CHB, registrar in pathology

D J GOLDIE, MD, MRCPATH, consultant chemical pathologist

University Department of Medicine, Southmead Hospital, Bristol BS10 5NB

M HARTOG, DM, FRCP, reader in medicine

A P ROBINSON, BSC, MRCP, medical registrar
}

patients using either Dextrostix or Reflotest strips and reading $\vec{N}$ them on Eyetone and Reflomat meters respectively. The merits of this approach are discussed below.

We have explored the possibility of measuring glucose in capillary blood samples spotted on to filter paper, dried, and subsequently eluted in the first stage of the assay. Such an approach was suggested in 1961,6 and several techniques have been described ${ }^{6-8}$; so far as we know, however, they are not widely used. We decided to investigate this approach again using, in particular, a common micro-glucose-oxidase method ${ }^{9}$ that is used routinely in our laboratory for paediatric investigations.

\section{Materials and methods}

Patients in general medical wards were asked to provide capillary blood samples by finger-prick using sterile lancets (Lance Blader Ltd). The drops obtained were applied gently to plain filter paper (Whatman No 4619) and allowed to soak through, giving a spot at least $6 \mathrm{~mm}$ diameter (ensured by viewing from both sides of the paper). If this size was not obtained another drop was applied immediately before any appreciable drying occurred. Up to six spots were obtained from each patient. Similar blood spots were made on the same piece of filter paper with venous blood withdrawn by venepuncture immediately after the patients had taken their capillary samples, and the remaining venous blood was added to a fluoride tube. The filter paper spots were dried and stored at room temperature.

The reagents used in the assays were as described by Trinder $^{9}$; D-glucose $10 \mathrm{mmol} / 1(180 \mathrm{mg} / 100 \mathrm{ml})$ in saturated benzoic acid served as standard.

\section{ASSAY PROCEDURE}

Whole blood glucose was assayed as described, ${ }^{9}$ except that $500 \mu l$ of the supernatant after protein precipitation was added to $1 \mathrm{ml}$ of colour reagent. A quality-control sample was assayed in each batch.

Blood spots-Protein precipitant $(500 \mu \mathrm{l})$ was added to a series of labelled test-tubes. For each sample one $6 \mathrm{~mm}$ diameter disc (containing approximately $11 \mu \mathrm{l}$ of blood) was punched from the centre of each of two blood spots (enabling the assay to be performed in duplicate). Similar discs were punched from the plain filter paper (as blanks). Punching was performed from the reverse side of the filter paper to which the blood had been applied to ensure completeness of the sample. Each disc was then added to a test-tube, capped, mixed by inversion, and left to elute for at least one hour at room temperature. The discs were then removed with forceps and the eluate centrifuged. Aliquots of the clear supernatants $(250 \mu \mathrm{l})$ were 